\title{
On the factorization of consecutive integers
}

\author{
M.A. Bennett, M. Filaseta and O. Trifonov
}

\section{Introduction}

A classical result of Sylvester [21] (see also [16], [17]), generalizing Bertrand's Postulate, states that the greatest prime divisor of a product of $k$ consecutive integers greater than $k$ exceeds $k$. More recent work in this vein, well surveyed in [18], has focussed on sharpening Sylvester's theorem, or upon providing lower bounds for the number of prime divisors of such a product. As noted in [18], a basic technique in these arguments is to make a careful distinction between "small" and "large" primes, and then apply sophisticated results from multiplicative number theory. Along these lines, if we write

$$
\left(\begin{array}{l}
n \\
k
\end{array}\right)=U \cdot V, \quad n \geq 2 k,
$$

where $U$ is composed only of primes $\leq k$ and $V$ only of those exceeding $k$, then Ecklund, Eggleton, Erdös and Selfridge [5] deduce the inequality $U<V$, valid with at most finitely many exceptions. To prove this, they employ a variety of combinatorial lemmata, the Prime Number Theorem, and a result of Mahler [11] from Diophantine approximation. In fact, they obtain something much more explicit, except in the cases $k \in\{3,5,7\}$, where the appeal to [11] leads to ineffectivity (rather than a precise list of exceptional pairs $(n, k)$ ). In this situation, we find in [5] a conjecture, described as the "most obvious outstanding problem" in this area, characterizing the exceptional pairs for $k \in\{3,5,7\}$ (there are believed to be precisely 19). This also appears as

The first author was partially supported by the Natural Sciences and Engineering Research Council of Canada, the second author by the National Science Foundation and the National Security Agency, and the third author by the National Science Foundation. 
problem B31 in [9] and is referenced in [6]. In this paper, as a byproduct of rather more general results (see Section 2), we will prove this conjecture in two of the three cases:

Theorem 1.1. If $k \in\{5,7\}, n \geq 2 k$ is an integer, and we write

$$
\left(\begin{array}{l}
n \\
k
\end{array}\right)=U \cdot V
$$

where $U$ and $V$ are integers with $P(U) \leq k$ and $V$ coprime to $k$ !, then it follows that $V>U$, unless

$$
(n, k) \in\{(10,5),(12,5),(21,7),(28,5),(30,7),(54,7)\} .
$$

Here, we denote by $P(m)$, the greatest prime factor of an integer $m$ (with $P( \pm 1)=1)$. Our approach is based upon careful examination of the case of two consecutive integers. As far back as 1897, C. Störmer [20] showed that the largest prime factor of $x^{2}+x$ tends to infinity with $x$ and gave an algorithm for finding every $x$ for which $x^{2}+x$ has all of its prime factors less than a prescribed bound. D. H. Lehmer [10], improving on this algorithm, determined explicitly the 869 positive integers $x$ for which the largest prime factor of $x^{2}+x$ is $\leq 41$. Fixing primes $p$ and $q$, for $x$ sufficiently large and for $k, l$, and $y$ nonnegative integers satisfying

$$
x^{2}+x=p^{k} q^{l} y,
$$

it follows from [20] that the value of $y$ is necessarily large. In fact, Mahler [11] applied a $p$-adic version of Roth's theorem from Diophantine approximation due to Ridout [13] to show that for an arbitrary $\varepsilon>0$ and $x$ sufficiently large (depending on $p, q$ and $\varepsilon$ ), the value of $y$ exceeds $x^{1-\varepsilon}$. Unfortunately, this result is ineffective in the sense that it is not possible to explicitly quantify the term "sufficiently large". The strongest effective analogue of this statement available to us, in general, is due to Stewart [19], who obtained an estimate of the shape $y \gg x^{\delta}$ for certain $\delta=\delta(p, q)>0$. This result is based on careful use of lower bounds for linear forms in logarithms and applies also to the situation where the right hand side of (1.1) contains an arbitrary fixed list of primes.

For the purpose of proving Theorem 1.1 and for other applications, however, we require a result that is valid for larger values of $\delta$ than those implicit in [19], yet still effective (indeed explicit). In this paper, we will deduce such estimates via the hypergeometric method of Thue and Siegel. We establish, by way of example, the following : 
Theorem 1.2. Let $x$ be a positive integer not in the set $\{1,2,3,8\}$, and suppose that $k, l$, and $y$ are nonnegative integers satisfying

$$
x^{2}+x=2^{k} 3^{l} y .
$$

Then $y>x^{0.285}$.

The need for a result of this flavour (a very special case of the statements of our Section 2) was actually the prime motivating force for this paper. Theorem 1.2 plays a crucial role in work of the last two authors and Travis Kidd (in preparation) on the irreducibility of $L_{m}^{(m)}(x)$, where

$$
L_{m}^{(\alpha)}(x)=\sum_{j=0}^{m} \frac{(m+\alpha)(m-1+\alpha) \cdots(j+1+\alpha)(-x)^{j}}{(m-j) ! j !}
$$

is the generalized Laguerre polynomial. In particular, it helps eliminate the possibility of $L_{m}^{(m)}(x)$ having a quadratic factor for $m>2$. This enables one to show that the polynomial $L_{m}^{(m)}(x)$ is irreducible for each integer $m>2$ with $m \equiv 2(\bmod 4)$, whence, following the work of I. Schur $[16,17]$ and R. Gow [8], there is, for every integer $m \geq 2$, a generalized Laguerre polynomial of degree $m$ having Galois group the alternating group $A_{m}$.

The layout of this paper is as follows. In Section 2, we provide notation and statements of our results in full generality. In Section 3, we introduce the approximating polynomials that will play the key role in our proofs (Padé approximants to the binomial function). In Section 4, we discuss needed bounds, archimedean estimates, on values of these approximating polynomials. In Section 5, we obtain nonarchimedean estimates associated with the polynomials; these correspond to bounds for the greatest common divisor of the coefficients associated with the approximating polynomials. In Section 6 , we give a computational argument for obtaining all small solutions associated with the diophantine inequalities in this paper. Sections 7 through 10 are devoted to completing the proofs of the main results in the paper.

Acknowledgments: The authors express their gratitude to Frits Beukers and Rob Tijdeman for early discussions that aided in their investigations. They are also grateful to Carrie Finch and Mark Kozek who read through an early version of the paper and suggested various changes. 


\section{The more general theorems}

We will deduce Theorems 1.1 and 1.2 as reasonably straightforward consequences of the following result.

Theorem 2.1. Let $p, q$ and $\lambda=\lambda(p, q)$ be as follows:

\begin{tabular}{|c|c|c||c|c|c|}
\hline$p$ & $q$ & $\lambda(p, q)$ & $p$ & $q$ & $\lambda(p, q)$ \\
\hline 2 & 3 & 0.285 & 3 & 11 & 0.329 \\
2 & 5 & 0.258 & 3 & 13 & 0.231 \\
2 & 7 & 0.259 & 5 & 7 & 0.227 \\
2 & 11 & 0.059 & 5 & 11 & 0.199 \\
2 & 13 & 0.054 & 5 & 13 & 0.163 \\
3 & 5 & 0.216 & 7 & 13 & 0.098 \\
3 & 7 & 0.038 & 11 & 13 & 0.037 \\
\hline
\end{tabular}

If $k, l, x_{1}$ and $x_{2}$ are nonnegative integers for which

$$
\left|p^{k} x_{1}-q^{l} x_{2}\right| \leq 100
$$

then either $p^{k} x_{1} \leq 1000$, one of $\left(p^{k} x_{1}, q^{l} x_{2}\right)$ and $\left(q^{l} x_{2}, p^{k} x_{1}\right)$ is in the set

$$
\begin{gathered}
\{(1024,972),(1029,1024),(1215,1210),(1280,1215),(1280,1250), \\
(1331,1250),(1372,1280),(1536,1458),(1792,1701),(2058,2048), \\
(2197,2187),(2500,2401),(2560,2500),(2673,2662),(3125,3072), \\
(3645,3584),(4394,4374),(5120,5103),(6591,6561),(6655,6561), \\
(7203,7168),(8019,7986),(8788,8748),(10985,10935),(13182,13122), \\
(14406,14336),(14641,14580),(15360,15309),(15379,15309), \\
(16038,15972),(17576,17496),(19773,19683),(21970,21870) \\
(24057,23958),(30618,30613),(32805,32768),(37268,37179), \\
(65610,65536),(190333,190269),(1771561,1771470)\},
\end{gathered}
$$

or

$$
\max \left\{x_{1}, x_{2}\right\}>\min \left\{p^{k} x_{1}, q^{l} x_{2}\right\}^{\lambda} .
$$

The reason that we have given these measures to three decimal places is that it is crucial for our arguments leading to Theorem 1.1 that we have

$$
\lambda(2,3)+\lambda(3,5)>1 / 2 \text {. }
$$


To achieve this requires extremely careful analysis and rather extensive computations; the measures for other values of $p$ and $q$ have not been computed nearly so carefully and hence may be readily sharpened. Note that we have restricted attention to prime values of $p$ and $q$. As our proof will reveal, this is only for simplicity and in light of our desired applications. There is no intrinsic reason for such a constraint. It is also worth noting that extending Theorem 1.1 to the case $k=3$ is essentially equivalent to sharpening Theorem 2.1, in the case $(p, q)=(2,3)$, to $\lambda(2,3)>1 / 2$. We suspect that proving such an inequality will require the introduction of fundamentally new ideas.

Theorem 2.1 will in fact follow from more general machinery. In order to state these results, we beg the reader's indulgence while we introduce some notation. Let $c>d \geq 1$ be integers and set $s=c / d$. We define

$$
\log L(s)=\frac{1}{d} \sum_{\substack{(c+d) / 2<j<c+d \\ r=j d^{-1} \bmod (c+d)}}\left(\Psi(f(c, d, r))-\Psi\left(\frac{r}{c+d}\right)\right),
$$

where

$$
f(c, d, r)= \begin{cases}\frac{1}{d}\left(1+\left\lfloor\frac{d r}{c+d}\right\rfloor\right) & \text { if } c \leq j<c+d \\ \frac{1}{c-d}\left(1+\left\lfloor\frac{(c-d) r}{c+d}\right\rfloor\right) & \text { if }(c+d) / 2<j<c .\end{cases}
$$

To clarify, the sum in the definition of $\log L(s)$ is over $j, \Psi(z)$ is the derivative of the logarithm of $\Gamma(z)$, and $r$ is chosen, in each case, to be the integer in $\{0,1, \ldots, c+d-1\}$ satisfying $r \equiv j d^{-1}(\bmod (c+d))$. For $0<z<1$, we take

$$
u_{1}=\frac{s(2-z)-\sqrt{s^{2} z^{2}+4-4 z}}{2(1-z)(s+1)}
$$

and

$$
u_{2}=\frac{s z+2-\sqrt{s^{2} z^{2}+4-4 z}}{2 z(s+1)} .
$$

Writing

$$
\alpha(s)=\frac{(s+1)^{s+1}}{(s-1)^{s-1}},
$$

set

$$
Q(s, z)=\alpha(s) u_{1}^{s-1}\left(1-u_{1}\right)\left(1-u_{1}+z u_{1}\right)
$$


and

$$
E(s, z)=\alpha(s) u_{2}\left(1-u_{2}\right)\left(1-z u_{2}\right)^{s-1} .
$$

The choices of $u_{1}$ and $u_{2}$ above and their appearance in the definition of $Q(s, z)$ and $E(s, z)$ are not arbitrary. The function $u^{s-1}(1-u)(1-u+z u)$ obtains its maximum for $u \in[0,1]$ at $u=u_{1}$, while the maximum of the function $u(1-u)(1-z u)^{s-1}$ for $u \in[0,1]$ occurs at $u=u_{2}$.

Our main result, at least from an effective, rather than explicit, viewpoint is the following.

Theorem 2.2. Let $p$ and $q$ be distinct integers. Suppose that there exist positive integers $a, b, k_{0}, l_{0}$ and $D_{0}$ such that

$$
a p^{k_{0}}-b q^{l_{0}}=D_{0}
$$

and write

$$
z_{0}=D_{0} /\left(a p^{k_{0}}\right), \mathcal{M}_{1}=\min \left\{p^{k_{0}}, q^{l_{0}}\right\} \text { and } \mathcal{M}_{2}=\max \left\{p^{k_{0}}, q^{l_{0}}\right\}
$$

Assume further that there exists a rational number s satisfying $1<s<1 / z_{0}$,

$$
\Omega_{1}:=\frac{p^{k_{0}(s-1)} L(s)}{a b^{s} Q\left(s, z_{0}\right)}>1,
$$

and

$$
\Omega_{2}:=\frac{\mathcal{M}_{1}^{s} L(s)}{\left(a p^{k_{0}}\right)^{s-1} D_{0}^{2} E\left(s, z_{0}\right)}>1 .
$$

If $D$ is a positive integer and $\epsilon>0$, then there exists an effectively computable constant $x_{0}=x_{0}(p, q, s, D, \epsilon)$ such that if $x_{1}, x_{2}, k$ and $l$ are nonnegative integers satisfying $p^{k} x_{1} \geq x_{0}$ and

$$
\left|p^{k} x_{1}-q^{l} x_{2}\right| \leq D
$$

then

$$
\max \left\{x_{1}, x_{2}\right\} \geq\left(p^{k} x_{1}\right)^{\lambda_{1}-\epsilon} \quad \text { where } \quad \lambda_{1}=\frac{\log \left(\Omega_{2}\right)}{\log \left(\mathcal{M}_{2}^{s} \Omega_{2}\right)} .
$$

As a corollary, we obtain the following effective, inexplicit version of Theorem 2.1. 
Corollary 2.3. Let $p, q$ and $\lambda_{2}=\lambda_{2}(p, q)$ be as follows:

\begin{tabular}{|c|c|c||c|c|c|}
\hline$p$ & $q$ & $\lambda_{2}(p, q)$ & $p$ & $q$ & $\lambda_{2}(p, q)$ \\
\hline 2 & 3 & 0.2921 & 3 & 11 & 0.3453 \\
2 & 5 & 0.2679 & 3 & 13 & 0.2372 \\
2 & 7 & 0.2757 & 5 & 7 & 0.2667 \\
2 & 11 & 0.0698 & 5 & 11 & 0.2091 \\
2 & 13 & 0.0798 & 5 & 13 & 0.1838 \\
3 & 5 & 0.2238 & 7 & 13 & 0.1243 \\
3 & 7 & 0.0505 & 11 & 13 & 0.0729 \\
\hline
\end{tabular}

If $D$ is a positive integer, then there exists an effectively computable constant $x_{0}=x_{0}(D)$ such that if $x_{1}, x_{2}, k$ and $l$ are nonnegative integers satisfying

$$
p^{k} x_{1} \geq x_{0} \text { and }\left|p^{k} x_{1}-q^{l} x_{2}\right| \leq D,
$$

then

$$
\max \left\{x_{1}, x_{2}\right\}>\left(p^{k} x_{1}\right)^{\lambda_{2}} .
$$

We note that we are not asserting that we can always apply Theorem 2.2 to obtain results of this nature for general $p$ and $q$. Indeed, we are apparently unable to derive anything of a nontrivial nature when $p=7$ and $q=11$.

To prove Theorem 2.1, we require a completely explicit version of Theorem 2.2. To state this, we need some further definitions. If $c>d \geq 1$ are integers, and $n=d m-\delta$ for $\delta \in\{0,1\}$, define

$$
\mathcal{G}(c, d, n)=\underset{r \in\{0,1, \ldots, n\}}{\operatorname{gcd}}\left(\left(\begin{array}{c}
2 n-r \\
n
\end{array}\right)\left(\begin{array}{c}
c m-n-1+r \\
r
\end{array}\right)\right) .
$$

Suppose that we have

$$
\mathcal{G}(c, d, n) \geq L_{1}(s)^{d m} \quad \text { for } \quad m>m_{0}=m_{0}\left(c, d, L_{1}(s)\right) .
$$

Note that we may trivially take $m_{0}=0$ and $L_{1}(s)=1$. In Section 5, we will see what stronger bounds are possible. Define

$$
C_{1, \delta}=\frac{\alpha(s)^{d}\left(s^{2}-1\right)^{(-1)^{\delta} / 2}}{2 \pi Q(s, z)^{d}} \cdot \int_{0}^{1} u^{c-d-1+\delta}(1-u)^{d-\delta}(1-u+z u)^{d-\delta} d u,
$$


and

$$
C_{2, \delta}=\frac{\alpha(s)^{d}\left(s^{2}-1\right)^{(-1)^{\delta} / 2}}{2 \pi E(s, z)^{d}} \cdot \int_{0}^{1} u^{d-\delta}(1-u)^{d-\delta}(1-z u)^{c-d-1+\delta} d u .
$$

Also, write

$$
\begin{gathered}
\Omega_{3}=\frac{p^{k_{0}(s-1)} L_{1}(s)}{a b^{s} Q\left(s, z_{0}\right)}, \quad \Omega_{4}=\frac{\mathcal{M}_{1}^{s} L_{1}(s)}{\left(a p^{k_{0}}\right)^{s-1} D_{0}^{2} E\left(s, z_{0}\right)}, \quad \lambda_{3}=\frac{\log \left(\Omega_{4}\right)}{\log \left(\mathcal{M}_{2}^{s} \Omega_{4}\right)}, \\
\kappa_{1}=\max _{\delta \in\{0,1\}} \frac{2 D C_{1, \delta}}{\left(a p^{k_{0}}\right)^{\delta}}, \quad \kappa_{2}=\min _{\delta \in\{0,1\}} \frac{\left(a p^{k_{0}}\right)^{1-\delta}}{2 D_{0}^{1-2 \delta} C_{2, \delta}},
\end{gathered}
$$

and, if $\epsilon>0$, set

$$
x_{0}=\mathcal{M}_{2}^{\frac{c(M+1)}{1-\lambda_{3}}},
$$

where

$$
M=\max \left\{\frac{\log \left(\kappa_{1}\right)}{d \log \Omega_{3}}, \frac{\left(1+\lambda_{3}\right) \log \left(\mathcal{M}_{2}^{c} \kappa_{2}^{-1}\right)}{\epsilon d \log \left(\mathcal{M}_{2}^{s} \Omega_{4}\right)}, \frac{\left(1+\lambda_{3}\right) \log \left(\kappa_{2}\right)}{\epsilon d \log \left(\mathcal{M}_{2}^{s} \Omega_{4}\right)}, m_{0}\right\} .
$$

We have

Theorem 2.4. Let $p$ and $q$ be distinct positive integers. Suppose that there exist positive integers $a, b, k_{0}, l_{0}$ and $D_{0}$ such that

$$
a p^{k_{0}}-b q^{l_{0}}=D_{0}
$$

and write

$$
z_{0}=D_{0} /\left(a p^{k_{0}}\right), \mathcal{M}_{1}=\min \left\{p^{k_{0}}, q^{l_{0}}\right\} \text { and } \mathcal{M}_{2}=\max \left\{p^{k_{0}}, q^{l_{0}}\right\} .
$$

Assume further that there exists a rational number $s$ with $1<s<1 / z_{0}, \Omega_{3}>$ 1 and $\Omega_{4}>1$. Then for all positive integers $D, x_{1}$ and $x_{2}$ and nonnegative integers $k$ and $l$ and for all $\epsilon>0$, if

$$
\min \left\{p^{k} x_{1}, q^{l} x_{2}\right\} \geq x_{0}
$$

and

$$
\left|p^{k} x_{1}-q^{l} x_{2}\right| \leq D
$$

then

$$
\max \left\{x_{1}, x_{2}\right\} \geq \min \left\{p^{k} x_{1}, q^{l} x_{1}\right\}^{\lambda_{3}-\epsilon}
$$




\section{Some useful polynomials}

Our results will require careful analysis of Padé approximants to the binomial function $(1-z)^{n}$. In the case that $n$ is a positive integer, these are just polynomials, which we may construct as follows. Let us suppose that $A, B$ and $C$ are positive integers and define

$$
\begin{gathered}
P_{A, B, C}(z)=\frac{(A+B+C+1) !}{A ! B ! C !} \int_{0}^{1} u^{A}(1-u)^{B}(z-u)^{C} d u, \\
Q_{A, B, C}(z)=\frac{(-1)^{C}(A+B+C+1) !}{A ! B ! C !} \int_{0}^{1} u^{B}(1-u)^{C}(1-u+z u)^{A} d u
\end{gathered}
$$

and

$$
E_{A, B, C}(z)=\frac{(A+B+C+1) !}{A ! B ! C !} \int_{0}^{1} u^{A}(1-u)^{C}(1-z u)^{B} d u .
$$

Arguing as in Section 2 of [2], we deduce that

$$
P_{A, B, C}(z)-(1-z)^{B+C+1} Q_{A, B, C}(z)=z^{A+C+1} E_{A, B, C}(z) .
$$

One may note, by comparison to e.g. Beukers [4], that if $A=C$, then $P_{A, B, C}(z)$ and $Q_{A, B, C}(z)$ correspond to the diagonal Padé approximants to $(1-z)^{B+C+1}$ with error term $E_{A, B, C}(z)$. The following results are given in [2] and [4] :

Lemma 3.1. The expressions $P_{A, B, C}(z), Q_{A, B, C}(z)$ and $E_{A, B, C}(z)$ satisfy

$$
\begin{aligned}
P_{A, B, C}(z) & =\sum_{r=0}^{C}\left(\begin{array}{c}
A+B+C+1 \\
r
\end{array}\right)\left(\begin{array}{c}
A+C-r \\
A
\end{array}\right)(-z)^{r}, \\
Q_{A, B, C}(z) & =(-1)^{C} \sum_{r=0}^{A}\left(\begin{array}{c}
A+C-r \\
C
\end{array}\right)\left(\begin{array}{c}
B+r \\
r
\end{array}\right) z^{r}
\end{aligned}
$$

and

$$
E_{A, B, C}(z)=\sum_{r=0}^{B}\left(\begin{array}{c}
A+r \\
r
\end{array}\right)\left(\begin{array}{c}
A+B+C+1 \\
A+C+r+1
\end{array}\right)(-z)^{r}
$$


Lemma 3.2. There is a non-zero integer $D=D(A, B)$ for which

$$
P_{A, B, A}(z) Q_{A+1, B-1, A+1}(z)-Q_{A, B, A}(z) P_{A+1, B-1, A+1}(z)=D z^{2 A+1} .
$$

In particular, Lemma 3.1 implies that $P_{A, B, C}(z), Q_{A, B, C}(z)$ and $E_{A, B, C}(z)$ are polynomials in $z$ with integer coefficients, and Lemma 3.2 implies that $P_{A, B, A}(z)$ and $P_{A+1, B-1, A+1}(z)$ (as well as $Q_{A, B, A}(z)$ and $Q_{A+1, B-1, A+1}(z)$ ) are relatively prime polynomials.

\section{Bounding the Approximants}

For our applications, we will have need of asymptotically sharp bounds for the polynomials defined in the preceding section, both in archimedean and nonarchimedean metrics. We take $c$ and $d$ to be relatively prime positive integers. As in (2.7), we suppose $c>d$ and that $n=d m$ or $d m-1$. Write $s=c / d$. Here and subsequently, let $A=C=n, B=c m-n-1$ and write, suppressing various dependencies,

$$
P_{n}(z)=P_{n, c m-n-1, n}(z), \quad Q_{n}(z)=Q_{n, c m-n-1, n}(z),
$$

and

$$
E_{n}(z)=E_{n, c m-n-1, n}(z)
$$

Our next result is essentially Lemma 5 of [2] and follows from replacing $-1 / N$ by $z$, and noting that the argument given there is still valid. We remark that some variable names have been modified.

Lemma 4.1. If $n=d m-\delta$ for $\delta \in\{0,1\}$, then

$$
\left|Q_{n}(z)\right|<C_{1, \delta}(Q(s, z))^{d m}
$$

and

$$
\left|E_{n}(z)\right|<C_{2, \delta}(E(s, z))^{d m} .
$$

For fixed values of $c, d, z$ and, hence, $s$, the values of $Q(s, z), E(s, z)$, and the integrals appearing in $C_{1, \delta}$ and $C_{2, \delta}$ of Lemma 4.1 can be computed exactly. We therefore can use Lemma 4.1 to determine bounds for $Q_{n}(z)$ and $E_{n}(z)$. While we will make no efforts to establish the fact, it is easy to show that these bounds are asymptotically sharp. 


\section{Nonarchimedean estimates}

We next turn our attention to nonarchimedean analogues of Lemma 4.1. Our main goal will be to derive strong lower bounds upon the quantity $\mathcal{G}(c, d, n)$ defined in (2.7). An implicit result of interest to us is the following

$$
\liminf _{m \rightarrow \infty} \mathcal{G}(c, d, n)^{1 /(d m)} \geq L(s) .
$$

Here, we assume that $n=d m$ or $d m-1$. In fact, with a little work, it is not difficult to deduce equality here and to replace the liminf with the limit (though, for our applications, this is relatively unimportant). To prove Theorem 2.1, we require more explicit estimates for $L_{1}(c / d)$ for certain fixed choices of integers $c$ and $d$; recall that this function provides a lower bound for $\mathcal{G}(c, d, n)$. We will prove the following :

Proposition 5.1. Inequality (2.8) holds for the values of $c, d, L_{1}(c / d)$ and $m_{0}$ indicated in the following table.

\begin{tabular}{|c|c|c|c||c|c|c|c|}
\hline$c$ & $d$ & $L_{1}(c / d)$ & $m_{0}$ & $c$ & $d$ & $L_{1}(c / d)$ & $m_{0}$ \\
\hline 9 & 8 & 1.1742 & 25 & 5 & 3 & 1.5454 & 86 \\
8 & 7 & 1.1951 & 28 & 3 & 1 & 1.5498 & 260 \\
7 & 6 & 1.2219 & 53 & 25 & 17 & 1.5540 & 582 \\
6 & 5 & 1.2581 & 35 & 7 & 4 & 1.6219 & 60 \\
5 & 4 & 1.3098 & 50 & 8 & 3 & 1.6560 & 149 \\
9 & 7 & 1.3317 & 15 & 9 & 5 & 1.6636 & 79 \\
7 & 5 & 1.4135 & 74 & 5 & 2 & 1.7017 & 231 \\
4 & 3 & 1.4170 & 153 & 7 & 3 & 1.7282 & 161 \\
3 & 2 & 1.5395 & 138 & 9 & 4 & 1.7666 & 87 \\
8 & 5 & 1.5407 & 53 & 2 & 1 & 1.9377 & 150 \\
\hline
\end{tabular}

Our chief tool in establishing Proposition 5.1 is the following result, Lemma 3 of [2]. Its form is suggested by the coefficients of the approximating polynomials.

Lemma 5.2. Suppose $t$ is a positive integer satisfying

$$
\left\lfloor\frac{A t}{A+B+C}\right\rfloor+\left\lfloor\frac{B t}{A+B+C}\right\rfloor+\left\lfloor\frac{C t}{A+B+C}\right\rfloor=t-2
$$

and that we define

$$
M(A, B, C, t)=\max \left\{\frac{A}{\left\lfloor\frac{A t}{A+B+C}\right\rfloor+1}, \frac{B}{\left\lfloor\frac{B t}{A+B+C}\right\rfloor+1}, \frac{C}{\left\lfloor\frac{C t}{A+B+C}\right\rfloor+1}\right\} .
$$


If $p$ is a prime for which

$$
M(A, B, C, t)<p \leq \frac{A+B+C}{t},
$$

then

$$
p \mid\left(\begin{array}{c}
A+C-r \\
C
\end{array}\right)\left(\begin{array}{c}
B+r \\
r
\end{array}\right) \text { for all } r \in\{0,1, \ldots, A\} .
$$

Recall that our goal is to obtain a lower bound for $\mathcal{G}(c, d, n)$. We begin by noting that the set of primes arising from Lemma 5.2 are disjoint for different values of $t$. To see this, observe that $(x+y) /(u+v)$ is between $x / u$ and $y / v$ for any positive real numbers $x, y, u$ and $v$. Set

$d_{1}=\left\lfloor\frac{A t}{A+B+C}\right\rfloor+1, d_{2}=\left\lfloor\frac{B t}{A+B+C}\right\rfloor+1$ and $d_{3}=\left\lfloor\frac{C t}{A+B+C}\right\rfloor+1$.

Then we have

$$
M(A, B, C, t)=\max \left\{\frac{A}{d_{1}}, \frac{B}{d_{2}}, \frac{C}{d_{3}}\right\} \geq \max \left\{\frac{A}{d_{1}}, \frac{B+C}{d_{2}+d_{3}}\right\} \geq \frac{A+B+C}{d_{1}+d_{2}+d_{3}} .
$$

From (5.2), we have $d_{1}+d_{2}+d_{3}=t+1$ and hence

$$
M(A, B, C, t) \geq \frac{A+B+C}{t+1},
$$

which implies our claim.

From Lemma 5.2, we thus have that

$$
\mathcal{G}(c, d, n) \geq \prod_{t}\left(\prod_{p} p\right)
$$

where $t$ ranges over all positive integers satisfying

$$
2\left\lfloor\frac{n t}{c m+n-1}\right\rfloor+\left\lfloor\frac{(c m-n-1) t}{c m+n-1}\right\rfloor=t-2
$$

and $p$ is prime with

$$
M(A, B, C, t)<p \leq \frac{c m+n-1}{t}
$$


(where $M(A, B, C, t)$ is as in (5.3), remembering that we take $A=C=n$ and $B=c m-n-1)$. With $x=n t /(c m+n-1)$, we can rewrite $(5.5)$ as $2\lfloor x\rfloor+\lfloor t-2 x\rfloor=t-2$. One checks that this is equivalent to

$$
\left\{\frac{n t}{c m+n-1}\right\}>\frac{1}{2}
$$

Suppose that $m \geq 2 t$ and $\{t /(s+1)\}>1 / 2$. We justify next that (5.6) holds so that if $m \geq 2 t$, then the first product in (5.4) can be taken over all $t$ for which $\{t /(s+1)\}>1 / 2$. To see that (5.6) holds, we begin with

$$
\frac{n t}{c m+n-1}>\frac{d t}{c+d}-\frac{1}{2(c+d)}=\frac{t}{s+1}-\frac{1}{2(c+d)} .
$$

This inequality can be easily verified by using $m \geq 2 t$ and $d m \leq n+1$, and the equality follows immediately from $s=c / d$. To establish (5.6) and also to help simplify our expression for the lower bound on the primes $p$ appearing in (5.4), we will show

$$
\left\lfloor\frac{n t}{c m+n-1}\right\rfloor=\left\lfloor\frac{t}{s+1}\right\rfloor \text { and }\left\lfloor\frac{(c m-n-1) t}{c m+n-1}\right\rfloor=\left\lfloor\frac{(s-1) t}{s+1}\right\rfloor .
$$

Observe that $\{t /(s+1)\}>1 / 2$ and (5.7) imply $\lfloor n t /(c m+n-1)\rfloor \geq\lfloor t /(s+1)\rfloor$. To obtain the reverse inequality and, hence, the first part of (5.8), we use that

$$
\left\{\frac{d t}{c+d}\right\}=\left\{\frac{t}{s+1}\right\}>\frac{1}{2}>0
$$

implies

$$
\frac{d t}{c+d} \leq\left\lfloor\frac{d t}{c+d}\right\rfloor+1-\frac{1}{c+d}
$$

Thus, the first equation in (5.8) will follow if we can show

$$
\frac{n t}{c m+n-1}<\frac{d t}{c+d}+\frac{1}{c+d} \text {. }
$$

From $n \leq d m$ and $n \geq 2 d t-1 \geq d t$, we obtain

$$
\begin{aligned}
\frac{n t}{c m+n-1} & \leq \frac{t}{s+1-\frac{1}{n}}=\frac{t}{s+1}+\frac{t}{n(s+1)\left(s+1-\frac{1}{n}\right)} \\
& \leq \frac{t}{s+1}+\frac{1}{d(s+1)\left(s+1-\frac{1}{n}\right)}<\frac{d t}{c+d}+\frac{1}{c+d} .
\end{aligned}
$$


Hence, the first equation in (5.8) holds. Since the inequality $\{t /(s+1)\}>1 / 2$ is strict, we deduce that

$$
\frac{t}{s+1}-\left\lfloor\frac{t}{s+1}\right\rfloor-\frac{1}{2}=\frac{d t}{c+d}-\left\lfloor\frac{t}{s+1}\right\rfloor-\frac{1}{2} \geq \frac{1}{2(c+d)} .
$$

Combining this inequality with (5.7) and the first equation in (5.8), we obtain (5.6). Since now both $\{t /(s+1)\}$ and $\{n t /(c m+n-1)\}$ exceed $1 / 2$, the second equation in (5.8) follows from the first as the second can be rewritten

$$
\left\lfloor t-\frac{2 n t}{c m+n-1}\right\rfloor=\left\lfloor t-\frac{2 t}{s+1}\right\rfloor .
$$

Before proceeding, we make some observations. First, it is not difficult to modify the above arguments to show that if $m>t$ and $3 d>c$, then $\{t /(s+1)\}>1 / 2$ still implies $\{n t /(c m+n-1)\}>1 / 2$. As the condition $3 d>c$ is satisfied for the choices of $c$ and $d$ we consider, this allows for an extension on the range of $m$ considered. Also, we note that the conditions $\{n t /(c m+n-1)\}>1 / 2$ and $\{t /(s+1)\}>1 / 2$ are not equivalent, even with $m \geq 2 t$. If $t \equiv 0(\bmod c+d)$ and $n=d m-1$, for example, then $\{t /(s+1)\}=$ 0 but $\{n t /(c m+n-1)\}>1 / 2$. Also, if $c \equiv d \equiv 1(\bmod 2), t \equiv(c+d) / 2$ $(\bmod c+d)$ and $n=d m$, then $\{t /(s+1)\}=1 / 2$ but $\{n t /(c m+n-1)\}>1 / 2$. These issues can help with the computations but did not in the end improve our theorems, so we do not address these matters further.

Proposition 5.3. Suppose that $m$ and $t$ are positive integers with $m \geq 2 t$, and that $n=d m$ or $n=d m-1$. Then $\mathcal{G}(c, d, n)$ is divisible by all primes $p$ satisfying

$$
\frac{n}{\left\lfloor\frac{t}{s+1}\right\rfloor+1}<p \leq \frac{c m+n-1}{t} \quad \text { if }\left\{\frac{t}{s+1}\right\}>\frac{s}{s+1}
$$

and

$$
\frac{c m-n-1}{\left\lfloor\frac{(s-1) t}{s+1}\right\rfloor+1}<p \leq \frac{c m+n-1}{t} \quad \text { if } \frac{1}{2}<\left\{\frac{t}{s+1}\right\}<\frac{s}{s+1}
$$

In the case that $\{t /(s+1)\}=s /(s+1)$, the first or second range on $p$ above holds depending on whether $n=d m$ or $n=d m-1$, respectively. 
Proof. Observe that the condition $c>d>0$ that we have imposed on $c$ and $d$ implies $s /(s+1)>1 / 2$. We have that $m \geq 2 t$ and need only consider the case that $\{t /(s+1)\}>1 / 2$. With $A=C=n$ and $B=c m-n-1,(5.8)$ simplifies (5.3) to

$$
M(A, B, C, t)=\max \left\{\frac{n}{\left\lfloor\frac{t}{s+1}\right\rfloor+1}, \frac{c m-n-1}{\left\lfloor\frac{(s-1) t}{s+1}\right\rfloor+1}\right\} .
$$

To aid with making use of $M(A, B, C, t)$, we show next that if $n=d m$, then

$$
\left\{\frac{t}{s+1}\right\} \geq \frac{s}{s+1} \Longrightarrow \frac{n}{\left\lfloor\frac{t}{s+1}\right\rfloor+1} \geq \frac{c m-n-1}{\left\lfloor\frac{(s-1) t}{s+1}\right\rfloor+1}
$$

and

$$
\frac{1}{2}<\left\{\frac{t}{s+1}\right\}<\frac{s}{s+1} \Longrightarrow \frac{n}{\left\lfloor\frac{t}{s+1}\right\rfloor+1}<\frac{c m-n-1}{\left\lfloor\frac{(s-1) t}{s+1}\right\rfloor+1} .
$$

Let $k=\lfloor t /(s+1)\rfloor$. Since $\{t /(s+1)\}>1 / 2$, we deduce that

$$
\frac{n}{\left\lfloor\frac{t}{s+1}\right\rfloor+1}=\frac{n}{k+1} \quad \text { and } \quad \frac{c m-n-1}{\left\lfloor\frac{(s-1) t}{s+1}\right\rfloor+1}=\frac{c m-n-1}{\left\lfloor t-\frac{2 t}{s+1}\right\rfloor+1}=\frac{c m-n-1}{t-2 k-1} .
$$

The second inequality in (5.9) is now easily seen to hold if and only if

$$
n(t-2 k-1) \geq(c m-n-1)(k+1)
$$

which simplifies to

$$
n t+k+1 \geq n k+c m k+c m \text {. }
$$

Multiplying through by $d$ and using $n=d m$, we obtain that the second inequality in (5.9) is equivalent to

$$
n d t+d k+d \geq n d k+c n k+c n .
$$

Set $\eta=\{t /(s+1)\}=\{d t /(c+d)\}$. Now, suppose the first inequality in (5.9) holds. Then $\eta \geq s /(s+1)=c /(c+d)$ so that

$$
\frac{d t}{c+d}=\frac{t}{s+1}=k+\eta \Longrightarrow d t \geq k(c+d)+c .
$$


With this lower bound on $d t$, one easily checks that (5.11) holds and (5.9) follows. Now, suppose the first set of inequalities in (5.10) holds. We want to show that the inequality in (5.11) is not true. In this case,

$$
\eta=\left\{\frac{d t}{c+d}\right\}<\frac{s}{s+1}=\frac{c}{c+d} \Longrightarrow \eta \leq \frac{c-1}{c+d} .
$$

Thus,

$$
d t=(k+\eta)(c+d) \leq\left(k+\frac{c-1}{c+d}\right)(c+d)=k(c+d)+c-1 .
$$

As we want to show (5.11) does not hold, it suffices to establish then that $n>d k+d$. Since

$$
n=d m \geq 2 d t>\max \{d, 2 k c+2 k d\} \geq d k+d,
$$

(5.10) follows.

In the case that $n=d m-1,(5.9)$ and (5.10) can be replaced by

$$
\left\{\frac{t}{s+1}\right\}>\frac{s}{s+1} \Longrightarrow \frac{n}{\left\lfloor\frac{t}{s+1}\right\rfloor+1}>\frac{c m-n-1}{\left\lfloor\frac{(s-1) t}{s+1}\right\rfloor+1}
$$

and

$$
\frac{1}{2}<\left\{\frac{t}{s+1}\right\} \leq \frac{s}{s+1} \Longrightarrow \frac{n}{\left\lfloor\frac{t}{s+1}\right\rfloor+1} \leq \frac{c m-n-1}{\left\lfloor\frac{(s-1) t}{s+1}\right\rfloor+1} .
$$

The analysis is similar to the above. With the notation as before, the second inequality in (5.12) is equivalent to $n t+k+1>n k+c m k+c m$. Since $n=d m-1$, this can be written as

$$
n d t+d k+d>n d k+c n k+c n+c k+c .
$$

The first inequality in (5.12) implies

$$
d t=(k+\eta)(c+d) \geq k(c+d)+c+1,
$$

while the first inequality in (5.13) yields

$$
d t=(k+\eta)(c+d) \leq k(c+d)+c .
$$


In regards to $(5.12)$, note that

$$
\begin{aligned}
n=d m-1 & \geq 2 d t-1=2(k+\eta)(c+d)-1 \\
& \geq(2 k+1)(c+d)>(k+1)(c-d) .
\end{aligned}
$$

These are sufficient to justify (5.12) and (5.13). Proposition 5.3 follows.

To understand the contribution of the primes described in Proposition 5.3, we need to examine the intervals in which they lie rather carefully. Generally, we are interested in the integers $t$ in the sets

$$
\begin{aligned}
& U_{1}=\left\{t:\left\{\frac{t}{s+1}\right\}>\frac{s}{s+1}\right\}, \\
& U_{2}=\left\{t:\left\{\frac{t}{s+1}\right\}=\frac{s}{s+1}\right\},
\end{aligned}
$$

and

$$
U_{3}=\left\{t: \frac{1}{2}<\left\{\frac{t}{s+1}\right\}<\frac{s}{s+1}\right\} .
$$

Note that $t /(s+1)=d t /(c+d)$ and $s /(s+1)=c /(c+d)$. Writing $t=$ $(c+d) w+r$ where $0 \leq r<c+d$, we deduce that $t \in U_{1}$ precisely when $r$ is of the form $j d^{-1} \bmod (c+d)$ (i.e., the integer in $[0, c+d)$ that is congruent to $j d^{-1}$ modulo $c+d$ ) where $c<j<c+d$. Similarly, $t \in U_{3}$ precisely when $r$ is of the form $j d^{-1} \bmod (c+d)$ where $(c+d) / 2<j<c$. One checks also that $t \in U_{2}$ precisely when $r$ is of the form $j d^{-1} \bmod (c+d)$ where $j=c$, which is equivalent to the case that $r=c+d-1$. Setting

$$
W=\left\lfloor\frac{m}{2(c+d)}\right\rfloor-1
$$

we deduce from Proposition 5.3 that

$$
\log \mathcal{G}(c, d, d m) \geq \sum_{w=0}^{W}\left(S_{1}+S_{2}\right)
$$

where

$$
S_{1}=\sum_{\substack{c \leq j<c+d \\ r \equiv j d^{-1} \bmod (c+d)}} \max \left\{\theta\left(\frac{(c+d) m-1}{(c+d) w+r}\right)-\theta\left(\frac{d m}{d w+1+\lfloor d r /(c+d)\rfloor}\right), 0\right\}
$$


and

$$
S_{2}=\sum_{\substack{(c+d) / 2<j<c \\ r \equiv j d^{-1} \bmod (c+d)}} \max \left\{\theta\left(\frac{(c+d) m-1}{(c+d) w+r}\right)-\theta\left(\frac{(c-d) m-1}{(c-d) w+1+\left\lfloor\frac{(c-d) r}{c+d}\right\rfloor}\right), 0\right\} .
$$

Here, $\theta(x)=\sum_{p \leq x} \log p$. Also, we have

$$
\log \mathcal{G}(c, d, d m-1) \geq \sum_{w=0}^{W}\left(S_{1}^{\prime}+S_{2}^{\prime}\right)
$$

where

$$
S_{1}^{\prime}=\sum_{\substack{c<j<c+d \\ r \equiv j d^{-1} \bmod (c+d)}} \max \left\{\theta\left(\frac{(c+d) m-2}{(c+d) w+r}\right)-\theta\left(\frac{d m-1}{d w+1+\lfloor d r /(c+d)\rfloor}\right), 0\right\}
$$

and

$$
S_{2}^{\prime}=\sum_{\substack{(c+d) / 2<j \leq c \\ r \equiv j d^{-1} \bmod (c+d)}} \max \left\{\theta\left(\frac{(c+d) m-2}{(c+d) w+r}\right)-\theta\left(\frac{(c-d) m}{(c-d) w+1+\left\lfloor\frac{(c-d) r}{c+d}\right\rfloor}\right), 0\right\} .
$$

It is not the case that all of the maxima above are needed, but the appearance of the maxima clarifies our approach. To estimate an expression of the form $\theta(x)-\theta(y)$ appearing above, we combine a lower bound for $\theta(x)$ with an upper bound for $\theta(y)$. The resulting lower bound for $\theta(x)-\theta(y)$ may be negative, and in this case we can appeal to $\max \{\theta(x)-\theta(y), 0\} \geq 0$.

For Theorem 2.2, where we do not require explicit constants, it suffices to note that, asymptotically, $\theta(x) \sim x$ whereby we may conclude from (5.14) (recalling the definition of $f(c, d, r)$ from the Introduction) that

$$
\liminf _{m \rightarrow \infty} \frac{1}{d m} \log \mathcal{G}(c, d, d m)
$$

is bounded below by

$$
\frac{1}{d} \sum_{\substack{(c+d) / 2<j<c+d \\ r=j d^{-1} \bmod (c+d)}} \sum_{w=0}^{\infty}\left(\frac{1}{w+r /(c+d)}-\frac{1}{w+f(c, d, r)}\right) .
$$


Arguing similarly for $\mathcal{G}(c, d, d m-1)$ and noting that

$$
\frac{1}{d}\left(1+\left\lfloor\frac{d r}{c+d}\right\rfloor\right)=\frac{1}{c-d}\left(1+\left\lfloor\frac{(c-d) r}{c+d}\right\rfloor\right)=1
$$

in case $j=c$ (whence $r=c+d-1$ ), we deduce inequality (5.1) upon applying the identity

$$
\Psi(f(c, d, r))-\Psi\left(\frac{r}{c+d}\right)=\sum_{w=0}^{\infty}\left(\frac{1}{w+r /(c+d)}-\frac{1}{w+f(c, d, r)}\right)
$$

We now turn our attention to the proof of Proposition 5.1. We will appeal to inequalities for $\theta(x)$ due to Rosser and Schoenfeld [14], Schoenfeld [15], Ramaré and Rumely [12] and the first author [3]. The following is a consequence of Theorems 1 and 2 from [12], and of the second table in Section 5 from [3]; we also add the bound $\theta(x)<x$ for $0<x \leq 10^{11}$ of Rosser and Schoenfeld mentioned above.

Lemma 5.4. For $1 \leq x \leq 10^{11}$, we have

$$
x-2.072 \sqrt{x}<\theta(x)<x .
$$

Furthermore, for $x \geq 10^{8}$, we have

$$
|\theta(x)-x| \leq 0.000213 x
$$

Finally, if $x \geq x_{0}$ then

$$
\theta(x)>x\left(1-\frac{1}{\beta \log x}\right)
$$

for $\beta$ and $x_{0}$ as follows:

\begin{tabular}{|cc|cc|cc|}
\hline$\beta$ & $x_{0}$ & $\beta$ & $x_{0}$ & $\beta$ & $x_{0}$ \\
\hline 6 & 8623 & 3 & 1429 & $7 / 5$ & 149 \\
5 & 5407 & $5 / 2$ & 809 & $9 / 7$ & 101 \\
$9 / 2$ & 3527 & $7 / 3$ & 599 & $7 / 6$ & 67 \\
4 & 3301 & 2 & 563 & $8 / 7$ & 59 \\
$7 / 2$ & 2657 & $9 / 5$ & 347 & 1 & 41 \\
$10 / 3$ & 1973 & $5 / 3$ & 227 & $4 / 5$ & 2 \\
\hline
\end{tabular}


This lemma allows us to compute lower and upper bounds for the values of $\theta(x)$ appearing in the sums in (5.14) and (5.15) (depending on the sizes of the arguments). Observe that for $m \geq 10^{10}$, one can take $\beta=6$ in the lower bound for $\theta(x)$ above for each argument given in the summands.

For fixed $c$ and $d$, the proof of Proposition 5.1 splits into four cases, depending on the size of $m$ (where $n=d m$ or $d m-1$ ). For small $m$ (say for $m \leq 1000)$, we explicitly compute $\mathcal{G}(c, d, n)$ for each of $n=d m$ or $d m-$ 1. Then for "medium-sized" $m$, typically $1000<m \leq 50000$, we apply inequalities (5.14) and (5.15) directly. The benefit of appealing to these bounds is that the intervals under consideration do not change greatly as the value of $m$ is slightly increased and hence if we are able to show, for example, that $\mathcal{G}(c, d, d m)$ exceeds $L_{1}(c / d)^{d m}$ by a reasonable margin, then we can deduce inequalities of the shape

$$
\mathcal{G}(c, d, d(m+i)) \geq L_{1}(c / d)^{d(m+i)}
$$

for $0 \leq i \leq m_{0}$, where $m_{0}$ is a computable positive constant. This computational approach is discussed in greater detail in the first author's papers [3] and [1] (in particular, see Section 7 of the latter), where it is termed "boot-strapping" (also, see the next section where a similar approach is used on $k$ ). In practice, it works well to reduce these intermediate calculations to a workable level. For larger values of $m$ (usually $m \geq 50000$ ), we turn to Lemma 5.4 (as mentioned earlier, it is convenient to consider $m<10^{10}$ and $m \geq 10^{10}$ separately). In all cases except $(c, d)=(4,3)$ and $(25,17)$, this leads immediately to Proposition 5.1. If $(c, d)=(4,3)$, we apply our boot-strapping for $1000 \leq m<210000$ and Lemma 5.4 for $m \geq 210000$. For $(c, d)=(25,17)$, we calculate $\mathcal{G}(c, d, n)$ explicitly for $m \leq 2000$, count primes in the intervals given by (5.14) and (5.15) for $2000<m<3.7 \times 10^{6}$, and appeal to Lemma 5.4 for $m \geq 3.7 \times 10^{6}$. The computation, carried out in Pari GP on a number of Sun workstations, was moderately lengthy. Full details are available from the authors on request.

\section{Computations for small integers}

The final ingredient we require before we proceed with the proofs of our main results is a computational method for handling "small" values of $p^{k} x_{1}$ and $q^{l} x_{2}$. In this section, we will describe how we employ such a method to prove the following: 
Proposition 6.1. For primes $p$ and $q$ with $2 \leq p<q \leq 13$, suppose $k, l, x_{1}$ and $x_{2}$ are nonnegative integers for which

$$
\left|p^{k} x_{1}-q^{l} x_{2}\right| \leq 100, \quad x_{1} x_{2} \leq \min \left\{p^{k} x_{1}, q^{l} x_{2}\right\}^{1 / 3}
$$

and

$$
10000<p^{k} x_{1}<\exp \left(10^{6}\right) .
$$

Then $\left(p^{k} x_{1}, q^{l} x_{2}\right)$ or $\left(q^{l} x_{2}, p^{k} x_{1}\right)$ must be among

(13182, 13122), (14641, 14580), (16807, 16767), (19773, 19683), (31250, 31213),

$(32805,32768),(65610,65536),(65625,65536),(1771561,1771470)$.

If the condition $x_{1} x_{2} \leq \min \left\{p^{k} x_{1}, q^{l} x_{2}\right\}^{1 / 3}$ is replaced by

$$
\max \left\{x_{1}, x_{2}\right\} \leq \min \left\{p^{k} x_{1}, q^{l} x_{2}\right\}^{1 / 3},
$$

then $\left(p^{k} x_{1}, q^{l} x_{2}\right)$ or $\left(q^{l} x_{2}, p^{k} x_{1}\right)$ belongs to a set containing 93 pairs. The following table indicates, for each choice of primes $p$ and $q$, the largest value of $p^{k} x_{1}$ in such a pair and the corresponding value of $\Delta=p^{k} x_{1}-q^{l} x_{2}$.

\begin{tabular}{|c|c|c|c||c|c|c|c|}
\hline$p$ & $q$ & $\max p^{k} x_{1}$ & $\Delta$ & $p$ & $q$ & $\max p^{k} x_{1}$ & $\Delta$ \\
\hline 2 & 3 & 65536 & -74 & 3 & 13 & 220548015 & -27 \\
2 & 5 & 13828096 & -29 & 5 & 7 & 134375 & -81 \\
2 & 7 & 39059456 & -12 & 5 & 11 & 10625 & -23 \\
2 & 11 & 60555264 & 88 & 5 & 13 & 2693359375 & -47 \\
2 & 13 & 208666624 & -42 & 7 & 11 & 453789 & -82 \\
3 & 5 & 5078214 & 89 & 7 & 13 & 76832 & -63 \\
3 & 7 & 1495908 & 85 & 11 & 13 & 1742279 & 58 \\
3 & 11 & 1771470 & -91 & & & & \\
\hline
\end{tabular}

Furthermore, for a fixed $p$ and $q$ as above, among all pairs $\left(p^{k} x_{1}, q^{l} x_{2}\right)$ satisfying the conditions imposed, the maximal value of $k$ and the maximal value of $l$ occur for the pair $\left(p^{k} x_{1}, p^{k} x_{1}-\Delta\right)$ indicated by the table with the exception of the prime pair $(p, q)=(5,7)$ where 6 is the maximal such $k$ occurring, for example, with the pair $(31250,31213)$. Also, if $|\Delta| \leq 6$, then $\left(p^{k} x_{1}, q^{l} x_{2}\right)$ or $\left(q^{l} x_{2}, p^{k} x_{1}\right)$ corresponds to one of the pairs

$$
\begin{gathered}
(13125,13122),(13312,13310),(21875,21870),(26624,26620), \\
(30618,30613),(69632,69629),(688128,688127), \\
(1376256,1376254),(9764867,9764864),(19529734,19529728) .
\end{gathered}
$$


Theorem 2.4 allows us to obtain that (2.11) has no solutions for $y<x^{\lambda-\epsilon}$ provided that $x \geq x_{0}$, where

$$
x=\min \left\{p^{k} x_{1}, q^{l} x_{2}\right\} \text { and } y=\max \left\{x_{1}, x_{2}\right\} .
$$

The condition that $x \geq x_{0}$ can be eliminated and replaced by a list of solutions to (2.11) after an appropriate computation. In particular, we are able to deduce Theorem 1.1 based on such computations. Given the size of the $x_{0}$ we encounter, the computations to find all solutions to (2.11) with $x<x_{0}$ require some care. We describe in this section the approach that we use to obtain these solutions.

For our purposes, we fix $z=10000, x_{0}=e^{10^{6}}$ and $\lambda=1 / 3$, but we discuss the approach in some generality as it can easily be modified for other situations where $z>0, x_{0}>0$ and $\lambda \in(0,1)$. Notably, some care is needed for $\lambda>1 / 3$. Fix $D>0$ and primes $p$ and $q$ as before (renaming if necessary to account for the sign of $D$ ). We describe how we can obtain all pairs $\left(p^{k} x_{1}, q^{l} x_{2}\right)$, with $k, l, x_{1}$ and $x_{2}$ positive integers, for which

$$
p^{k} x_{1}-q^{l} x_{2}=D
$$

where

$$
z<x=q^{l} x_{2} \leq x_{0} \text { and } y=\max \left\{x_{1}, x_{2}\right\} \leq x^{\lambda} .
$$

Note that $D>0$ and (6.1) imply $q^{l} x_{2}=\min \left\{p^{k} x_{1}, q^{l} x_{2}\right\}$. The choice $z=$ 10000 is used for convenience and chosen so that the solutions to (6.1) with $x \leq z$ and $y \leq x^{\lambda}$, can be determined by a direct computation.

From the fact that $y \leq x^{\lambda}$, we have the inequality $x_{1} \leq\left(p^{k} x_{1}\right)^{\lambda}$ whereby

$$
x_{1} \leq\left(p^{k}\right)^{\lambda /(1-\lambda)}
$$

Hence,

$$
\left(p^{k}\right)^{1 /(1-\lambda)}=p^{k}\left(p^{k}\right)^{\lambda /(1-\lambda)} \geq p^{k} x_{1} \geq x>z .
$$

We deduce a lower bound on $k$ from the above and an upper bound on $k$ from (6.2), namely

$$
\left\lceil\frac{\log \left(z^{1-\lambda}\right)}{\log p}\right\rceil \leq k \leq\left\lfloor\frac{\log \left(x_{0}+D\right)}{\log p}\right\rfloor .
$$


We will consider an interval of $k$ simultaneously. Specifically, we consider $k \in\left[K, K+K^{\prime}\right)$ where

$$
\left\lceil\frac{\log \left(z^{1-\lambda}\right)}{\log p}\right\rceil \leq K<K+K^{\prime} \leq\left\lfloor\frac{\log \left(x_{0}+D\right)}{\log p}\right\rfloor+1 .
$$

For a given $K$ between the upper and lower limits above, we will define a positive integer $K^{\prime}$ in such a way that we dispose of the cases with $k \in$ $\left[K, K+K^{\prime}\right)$ all at once. Our main interest in considering an interval of $k$ is to speed up computations for large $k$. With this in mind, we consider $K^{\prime}=1$ for $K<200$ and will consider larger $K^{\prime}$ only in the case that $K \geq 200$.

An argument similar to the above gives

$$
x_{2} \leq\left(q^{l}\right)^{\lambda /(1-\lambda)} \text { and }\left\lceil\frac{\log \left(z^{1-\lambda}\right)}{\log q}\right\rceil \leq l \leq\left\lfloor\frac{\log x_{0}}{\log q}\right\rfloor .
$$

We will also want a second lower bound on $l$ obtained in an analogous manner. Observe that

$$
\left(q^{l}\right)^{1 /(1-\lambda)}=q^{l}\left(q^{l}\right)^{\lambda /(1-\lambda)} \geq q^{l} x_{2}=p^{k} x_{1}-D \geq \max \left\{q, p^{k}-D\right\} .
$$

For $k \in\left[K, K+K^{\prime}\right)$, we deduce

$$
l \geq\left\lceil\frac{(1-\lambda) \log \max \left\{q, p^{K}-D\right\}}{\log q}\right\rceil,
$$

whence

$$
l \geq B=\max \left\{\left\lceil\frac{(1-\lambda) \log \max \left\{q, p^{K}-D\right\}}{\log q}\right\rceil,\left\lceil\frac{\log \left(z^{1-\lambda}\right)}{\log q}\right\rceil\right\} .
$$

We explain now how the above lower bound on $l$ is enough to allow us to make efficient computations for obtaining the solutions to (6.1) and (6.2) for the main applications in this paper. As $k \in\left[K, K+K^{\prime}\right)$, there is a nonnegative integer $u<K^{\prime}$ such that $k=K+u$. From (6.1) and $l \geq B$, we deduce

$$
p^{u} x_{1} \equiv D p^{-K} \quad\left(\bmod q^{B}\right)
$$

For fixed $K$, we compute $B$ and the least positive integer solution to $x_{1}^{\prime} \equiv$ $D p^{-K}\left(\bmod q^{B}\right)$. Thus, $p^{u} x_{1} \equiv x_{1}^{\prime}\left(\bmod q^{B}\right)$. Recall that $K^{\prime}=1$ if $K<$ 
200. For these $K$, we have $u=0$. As $p, k=K$ and $D$ are known, we can check directly if $x_{1}=x_{1}^{\prime}$ provides us with a solution to (6.1) and (6.2).

Still with $K<200$, we justify next that there are no solutions to (6.1) and (6.2) with $x_{1}>x_{1}^{\prime}$. Here, $k=K$. A solution to (6.1) and (6.2), with $x_{1}>x_{1}^{\prime}$, necessarily satisfies $x_{1} \geq x_{1}^{\prime}+q^{B} \geq q^{B}$. By the definition of $B$, we have

$$
q^{B} \geq\left(p^{k}-D\right)^{1-\lambda}
$$

and hence, via (6.3),

$$
\left(p^{k}-D\right)^{1-\lambda} \leq\left(p^{k}\right)^{\lambda /(1-\lambda)}
$$

Observe that the maximum value of $\lambda /(1-\lambda)^{2}$ for $\lambda \in[0,1 / 3]$ is $3 / 4$. As $D \leq 100$, one checks that (6.6) implies that $p^{k}<141$. For $p$ as in Proposition 6.1 , we deduce $p^{k} \leq 128$. As $z=10^{4}$, we obtain from $128 x_{1} \geq p^{k} x_{1}>z$ that

$$
x_{1}^{2}>128 \geq p^{k} \Longrightarrow x_{1}^{3}>p^{k} x_{1} \Longrightarrow x_{1}>\left(p^{k} x_{1}\right)^{1 / 3}>x^{1 / 3} .
$$

Since $\lambda=1 / 3$, we see that (6.2) cannot hold.

For $K \geq 200$, we verify that there are no solutions to (6.1) and (6.2) for all $k \in\left[K, K+K^{\prime}\right)$ as follows. Here, we have $k=K+u$ with $u \in\left[0, K^{\prime}\right)$ and $p^{u} x_{1} \equiv x_{1}^{\prime}\left(\bmod q^{B}\right)$. Recall that $x_{1}^{\prime}$ is the minimal positive integer for which this congruence holds. It follows that $x_{1} \geq x_{1}^{\prime} / p^{u}$; otherwise, $p^{u} x_{1}$ would be too small for the congruence to hold. Given (6.3), we will be done justifying there are no solutions to (6.1) and (6.2) for $k \in\left[K, K+K^{\prime}\right)$ if we can show

$$
\frac{x_{1}^{\prime}}{p^{u}}>\left(p^{K+u}\right)^{\lambda /(1-\lambda)} \quad \text { for } u \in\left[0, K^{\prime}\right) .
$$

For $\lambda=1 / 3$, the exponent $\lambda /(1-\lambda)$ is $1 / 2$. It therefore suffices to establish that

$$
x_{1}^{\prime} \geq p^{K^{\prime}}\left(p^{K+K^{\prime}}\right)^{1 / 2} .
$$

Observe that the definition of $x_{1}^{\prime}$ implies that we can "expect" that $x_{1}^{\prime}$ is on the order of $q^{B}$ and that this is on the order of $p^{(1-\lambda) K}=p^{2 K / 3}$ or more. It follows that we should be able to take $K^{\prime}$ to be about $K / 9$. As $x_{1}^{\prime}$ and $K$ are known quantities in (6.7), to assure that (6.7) holds, we set

$$
K^{\prime}=\left\lfloor\frac{1}{3}\left(\frac{2 \log x_{1}^{\prime}}{\log p}-K\right)\right\rfloor .
$$


In our computations, $x_{1}^{\prime}$ is typically large enough that even a direct computation of $K^{\prime}$ via this simple formula takes more time than is feasible. We alleviated this problem by again taking advantage of the fact that $x_{1}^{\prime}$ should be near $q^{B}$. Beginning with $\lfloor B \log q / \log p\rfloor$ and decrementing by 1 as needed, one very quickly comes to an integer $w$ for which $p^{w}>x_{1}^{\prime}$ and this $w$ can be used in place of $\log x_{1}^{\prime} / \log p$ in the formula for $K^{\prime}$. Although we expect $K^{\prime}$ to be about $K / 9$, it is possible that $K^{\prime} \leq 0$. The idea then is to obtain $K^{\prime}$ as above and check that $K^{\prime}>0$. If so, then we can eliminate the possibility that (6.1) and (6.2) hold for all $k \in\left[K, K+K^{\prime}\right.$ ) simultaneously. Our checks showed in fact that $K^{\prime}>0$ in every case. In other words, as we progressed through the $K \geq 200$ eliminating intervals $\left[K, K+K^{\prime}\right)$ as we proceeded, the value of $K^{\prime}$ remained positive.

The above discussion illuminates the main parts of our algorithm for verifying Proposition 6.1. Given the above, the computations were straight forward and done with Maple 9.5.

\section{Proofs of Theorem 2.2 and Theorem 2.4}

We give the proof of Theorem 2.4. The proof of Theorem 2.2 proceeds along identical lines, only with $L_{1}(s)$ replaced by $L(s)^{1-\epsilon^{\prime}}$ for suitably small $\epsilon^{\prime}$, and $\Omega_{3}, \Omega_{4}$ and $\lambda_{3}$ replaced by $\Omega_{1}, \Omega_{2}$ and $\lambda_{1}$, respectively.

Let us begin by supposing that $p, q, k, l, x_{1}, x_{2}$ and $D$ satisfy (2.10) and (2.11). We assume throughout, as we may, that $x_{1} x_{2}$ is coprime to $p q$ and, renaming if necessary, suppose that

$$
p^{k} x_{1}>q^{l} x_{2} .
$$

For the duration of this proof, we employ the shorthand

$$
x=\min \left\{p^{k} x_{1}, q^{l} x_{2}\right\}=q^{l} x_{2} \text { and } y=\max \left\{x_{1}, x_{2}\right\} .
$$

Recall that $s=c / d$ where $c$ and $d$ are relatively prime positive integers. Set

$$
\begin{gathered}
m_{1}=\left\lfloor\frac{k}{k_{0} c}\right\rfloor, \quad m_{2}=\left\lfloor\frac{l}{l_{0} c}\right\rfloor, \\
\alpha=k-k_{0} c m_{1} \in\left[0, k_{0} c\right) \text { and } \beta=l-l_{0} c m_{2} \in\left[0, l_{0} c\right) .
\end{gathered}
$$

Then we may rewrite (2.11) in the form

$$
0<p^{k_{0} c m_{1}} x_{1}^{\prime}-q^{l_{0} c m_{2}} x_{2}^{\prime} \leq D
$$


where

$$
x_{1}^{\prime}=p^{\alpha} x_{1} \quad \text { and } \quad x_{2}^{\prime}=q^{\beta} x_{2} .
$$

Choosing $m=\min \left\{m_{1}, m_{2}\right\}$, we have

$$
0<p^{k_{0} c m} x_{1}^{\prime \prime}-q^{l_{0} c m} x_{2}^{\prime \prime} \leq D,
$$

where either $x_{1}^{\prime \prime}=x_{1}^{\prime}$ or $x_{2}^{\prime \prime}=x_{2}^{\prime}$.

Recall that $A=C=n$ and $B=c m-n-1$, where $n=d m$ or $d m-1$. The definition of $\mathcal{G}(c, d, n)$ in (2.7) then corresponds to the greatest common divisor of the coefficients of $Q_{n}(z)$ given in Lemma 3.1. Clearly, $\mathcal{G}(c, d, n)^{-1}$. $Q_{n}(z)$ is a polynomial with integer coefficients. Also, from Lemma 3.1, the degree of $P_{n}(z)$ is $n$. On the right-hand side of (3.4), the coefficient of $z^{j}$ is 0 for each $j \leq n$. We deduce then that $\mathcal{G}(c, d, n)^{-1} \cdot P_{n}(z)$ and, hence, $\mathcal{G}(c, d, n)^{-1} \cdot E_{n}(z)$ are also polynomials with integer coefficients.

Fixing once and for all $z=z_{0}=D_{0} /\left(a p^{k_{0}}\right)$ and substituting this into (3.4), we find that

$$
\left(a p^{k_{0}}\right)^{c m} P-\left(b q^{l_{0}}\right)^{c m} Q=E
$$

where

$$
P=\frac{1}{\mathcal{G}(c, d, n)}\left(a p^{k_{0}}\right)^{n} P_{n}\left(z_{0}\right), \quad Q=\frac{1}{\mathcal{G}(c, d, n)}\left(a p^{k_{0}}\right)^{n} Q_{n}\left(z_{0}\right)
$$

and

$$
E=\frac{1}{\mathcal{G}(c, d, n)}\left(a p^{k_{0}}\right)^{c m-n-1} D_{0}^{2 n+1} E_{n}\left(z_{0}\right)
$$

Multiplying (7.2) by $b^{c m} Q$ and (7.3) by $x_{2}^{\prime \prime}$, we deduce that

$$
p^{k_{0} c m}\left|b^{c m} Q x_{1}^{\prime \prime}-a^{c m} P x_{2}^{\prime \prime}\right| \leq b^{c m} D|Q|+|E| x_{2}^{\prime \prime} .
$$

From Lemma 3.2, it is straightforward to show that, for at least one of $n=d m$ and $d m-1$, we have $b^{c m} Q x_{1}^{\prime \prime} \neq a^{c m} P x_{2}^{\prime \prime}$. If not, then

$$
b^{c m} Q_{d m}\left(z_{0}\right) x_{1}^{\prime \prime}=a^{c m} P_{d m}\left(z_{0}\right) x_{2}^{\prime \prime} \text { and } b^{c m} Q_{d m-1}\left(z_{0}\right) x_{1}^{\prime \prime}=a^{c m} P_{d m-1}\left(z_{0}\right) x_{2}^{\prime \prime} .
$$

Multiplying the first of these equations by $P_{d m-1}\left(z_{0}\right)$ and the second by $P_{d m}\left(z_{0}\right)$, we find that

$$
Q_{d m}\left(z_{0}\right) P_{d m-1}\left(z_{0}\right)-Q_{d m-1}\left(z_{0}\right) P_{d m}\left(z_{0}\right)=0
$$


contradicting Lemma 3.2, since $z_{0} \neq 0$. Fixing $n=d m-\delta$, with $\delta \in\{0,1\}$ and $b^{c m} Q x_{1}^{\prime \prime} \neq a^{c m} P x_{2}^{\prime \prime}$, we deduce that

$$
p^{k_{0} c m} \leq b^{c m} D|Q|+|E| x_{2}^{\prime \prime}
$$

The idea is to show that each of $|Q|$ and $|E|$ is not too large, whereby we may employ (7.4) to obtain a lower bound on $x_{2}^{\prime \prime}$ (and hence on $y$ ).

Recall that we aim to show that $y \geq x^{\lambda_{3}-\epsilon}$. We may therefore suppose $y<x^{\lambda_{3}}$. With this restriction, we begin by demonstrating that

$$
d m>\max \left\{\frac{\log \left(\kappa_{1}\right)}{\log \Omega_{3}}, \frac{\left(1+\lambda_{3}\right) \log \left(\mathcal{M}_{2}^{c} \kappa_{2}^{-1}\right)}{\epsilon \log \left(\mathcal{M}_{2}^{s} \Omega_{4}\right)}, \frac{\left(1+\lambda_{3}\right) \log \left(\kappa_{2}\right)}{\epsilon \log \left(\mathcal{M}_{2}^{s} \Omega_{4}\right)}, d m_{0}\right\}
$$

Since

$$
y=\max \left\{p^{-\alpha} x_{1}^{\prime}, q^{-\beta} x_{2}^{\prime}\right\}<x^{\lambda_{3}}
$$

and either $x_{1}^{\prime \prime}=x_{1}^{\prime}$ or $x_{2}^{\prime \prime}=x_{2}^{\prime}$, it follows that

$$
\min \left\{p^{-\alpha} x_{1}^{\prime \prime}, q^{-\beta} x_{2}^{\prime \prime}\right\}<x^{\lambda_{3}} .
$$

Thus, either

$$
x=q^{l_{0} c m} x_{2}^{\prime \prime}=q^{l_{0} c m} q^{\beta} q^{-\beta} x_{2}^{\prime \prime}<q^{l_{0} c m} q^{l_{0} c} x^{\lambda_{3}}
$$

so that

$$
q^{l_{0} c m}>x^{1-\lambda_{3}} q^{-l_{0} c},
$$

or, if $x_{1}^{\prime \prime}=x_{1}^{\prime}$, arguing similarly,

$$
p^{k_{0} c m}>x^{1-\lambda_{3}} p^{-k_{0} c}
$$

These are equivalent to

$$
m>\frac{\left(1-\lambda_{3}\right) \log x-\log \left(q^{l_{0} c}\right)}{\log \left(q^{l_{0} c}\right)}
$$

and

$$
m>\frac{\left(1-\lambda_{3}\right) \log x-\log \left(p^{k_{0} c}\right)}{\log \left(p^{k_{0} c}\right)},
$$

respectively. The condition $x \geq x_{0}$ is equivalent to

$$
\left(1-\lambda_{3}\right) \log x \geq(M+1) \log \left(\mathcal{M}_{2}^{c}\right)
$$


and hence we have that $m>M$, whereby inequality (7.5) follows immediately.

Applying Lemma 4.1 and inequality (2.8), yields the upper bound

$$
|Q|<\frac{\left(a p^{k_{0}}\right)^{d m-\delta} C_{1, \delta} Q\left(s, z_{0}\right)^{d m}}{L_{1}(s)^{d m}} \leq \frac{\kappa_{1}}{2 D}\left(\frac{a p^{k_{0}} Q\left(s, z_{0}\right)}{L_{1}(s)}\right)^{d m} .
$$

Since (7.5) implies that $\kappa_{1}<\Omega_{3}^{d m}$, we may conclude from (7.4) that

$$
x_{2}^{\prime \prime}>\frac{p^{k_{0} c m}}{2|E|} .
$$

Since Lemma 4.1 leads to the inequality

$$
|E|<\frac{\left(a p^{k_{0}}\right)^{(c-d) m+\delta-1} D_{0}^{2 d m+1-2 \delta} C_{2, \delta} E\left(s, z_{0}\right)^{d m}}{L_{1}(s)^{d m}},
$$

it therefore follows that

$$
x_{2}^{\prime \prime}>\kappa_{2}\left(\frac{p^{k_{0}} L_{1}(s)}{a^{s-1} D_{0}^{2} E\left(s, z_{0}\right)}\right)^{d m}=\kappa_{2} \Omega_{4}^{d m}\left(\frac{p^{k_{0}}}{\mathcal{M}_{1}}\right)^{c m} \geq \kappa_{2} \Omega_{4}^{d m} .
$$

Since also

$$
x_{1}^{\prime \prime}>\left(\frac{q^{l_{0}}}{p^{k_{0}}}\right)^{c m} x_{2}^{\prime \prime}>\kappa_{2} \Omega_{4}^{d m}\left(\frac{q^{l_{0}}}{\mathcal{M}_{1}}\right)^{c m} \geq \kappa_{2} \Omega_{4}^{d m},
$$

we may conclude that

$$
y>\mathcal{M}_{2}^{-c} \min \left\{x_{1}^{\prime \prime}, x_{2}^{\prime \prime}\right\}>\mathcal{M}_{2}^{-c} \kappa_{2} \Omega_{4}^{d m} .
$$

From

$$
x=q^{l_{0} c m} x_{2}^{\prime \prime}<p^{k_{0} c m} x_{1}^{\prime \prime},
$$

we may thereby write

$$
\frac{\log y}{\log x} \geq \frac{-\log \left(\mathcal{M}_{2}^{c}\right)+\min \left\{\log x_{1}^{\prime \prime}, \log x_{2}^{\prime \prime}\right\}}{d m \log \left(\mathcal{M}_{2}^{s}\right)+\min \left\{\log x_{1}^{\prime \prime}, \log x_{2}^{\prime \prime}\right\}} .
$$

If $u$ and $v$ are positive numbers, then the function $(w-u) /(w+v)$ increases with $w$. It follows therefore that

$$
\frac{\log y}{\log x} \geq \frac{-\log \left(\mathcal{M}_{2}^{c}\right)+\log \left(\kappa_{2} \Omega_{4}^{d m}\right)}{d m \log \left(\mathcal{M}_{2}^{s}\right)+\log \left(\kappa_{2} \Omega_{4}^{d m}\right)}
$$


and hence $y \geq x^{\theta}$, where

$$
\theta=\frac{\log \left(\Omega_{4}\right)-\log \left(\mathcal{M}_{2}^{c} \kappa_{2}^{-1}\right) /(d m)}{\log \left(\mathcal{M}_{2}^{s} \Omega_{4}\right)+\log \left(\kappa_{2}\right) /(d m)} .
$$

From (7.5), we have

$$
\max \left\{\frac{1}{d m} \log \left(\mathcal{M}_{2}^{c} \kappa_{2}^{-1}\right), \frac{1}{d m} \log \left(\kappa_{2}\right)\right\}<\frac{\epsilon}{1+\lambda_{3}} \cdot \log \left(\mathcal{M}_{2}^{s} \Omega_{4}\right) .
$$

Using also $\lambda_{3}=\log \left(\Omega_{4}\right) / \log \left(\mathcal{M}_{2}^{s} \Omega_{4}\right)$, we deduce that

$$
\theta>\frac{\lambda_{3}-\frac{\epsilon}{1+\lambda_{3}}}{1+\frac{\epsilon}{1+\lambda_{3}}}>\lambda_{3}-\epsilon .
$$

This completes the proof of Theorem 2.4. As noted previously, Theorem 2.2 follows with minor modifications.

\section{Proofs of Corollary 2.3 and Theorem 2.1}

To prove Corollary 2.3, we apply Theorem 2.2, choosing parameters in the following fashion :

\begin{tabular}{|c|c|c|c|c|c|c|c|c|c|c|c|}
\hline$\{p, q\}$ & $a$ & $p^{k_{0}}$ & $b$ & $q^{l_{0}}$ & $s$ & $\{p, q\}$ & $a$ & $p^{k_{0}}$ & $b$ & $q^{l_{0}}$ & $s$ \\
\hline$\{2,3\}$ & 1 & 9 & 1 & 8 & 1.44829 & $\{3,11\}$ & 1 & 243 & 2 & 121 & 1.35497 \\
$\{2,5\}$ & 1 & 128 & 1 & 125 & 1.22744 & $\{3,13\}$ & 1 & 2197 & 1 & 2187 & 1.15161 \\
$\{2,7\}$ & 1 & 8 & 1 & 7 & 1.46706 & $\{5,7\}$ & 2 & 25 & 1 & 49 & 1.49683 \\
$\{2,11\}$ & 1 & 128 & 1 & 121 & 1.22806 & $\{5,11\}$ & 1 & 125 & 1 & 121 & 1.22853 \\
$\{2,13\}$ & 1 & 512 & 3 & 169 & 1.40712 & $\{5,13\}$ & 2 & 13 & 1 & 25 & 1.62743 \\
$\{3,5\}$ & 1 & 27 & 1 & 25 & 1.31653 & $\{7,13\}$ & 1 & 343 & 2 & 169 & 1.33134 \\
$\{3,7\}$ & 5 & 49 & 1 & 243 & 1.64610 & $\{11,13\}$ & 1 & 13 & 1 & 11 & 1.40106 \\
\hline
\end{tabular}

Observe that in applying Theorem 2.2, we take $c$ and $d$ to be relatively prime integers for which $s=c / d$ in the above table. As far as we are aware, these choices are essentially optimal to maximize the measures $\lambda_{2}(p, q)$ in Corollary 2.3. We know of no values of $a, b, k_{0}, l_{0}$ and $s$ which leads to a nontrivial result in case, for example, $(p, q)=(7,11)$. 
To prove Theorem 2.1, we utilize the same parameters $a, b, k_{0}$ and $l_{0}$, and take $D=100$. We further choose $s=c / d$ and $\epsilon$ as in the following table. In each case, we have the inequality

$$
\lambda_{3}(p, q)>\lambda(p, q)+\epsilon
$$

and are able to conclude that (2.2) holds, at least provided $x=\min \left\{p^{k} x_{1}, q^{l} x_{2}\right\}$ is suitably large, say $x \geq x_{0}$ for the stated values of $x_{0}$.

\begin{tabular}{|l|c|c|c|c|c|c|c|c|c|}
\hline$\{p, q\}$ & $c$ & $d$ & $\epsilon$ & $\log \left(x_{0}\right)$ & $\{p, q\}$ & $c$ & $d$ & $\epsilon$ & $\log \left(x_{0}\right)$ \\
\hline$\{2,3\}$ & 25 & 17 & 0.000099 & 695411 & $\{3,11\}$ & 7 & 5 & 0.0006 & 83857 \\
$\{2,5\}$ & 5 & 4 & 0.0003 & 96124 & $\{3,13\}$ & 7 & 6 & 0.001 & 63244 \\
$\{2,7\}$ & 3 & 2 & 0.0002 & 37549 & $\{5,7\}$ & 8 & 5 & 0.0007 & 53104 \\
$\{2,11\}$ & 5 & 4 & 0.0002 & 116863 & $\{5,11\}$ & 5 & 4 & 0.0004 & 67508 \\
$\{2,13\}$ & 3 & 2 & 0.0005 & 35516 & $\{5,13\}$ & 5 & 3 & 0.0008 & 22453 \\
$\{3,5\}$ & 4 & 3 & 0.00004 & 371785 & $\{7,13\}$ & 7 & 5 & 0.0007 & 60660 \\
$\{3,7\}$ & 5 & 3 & 0.0009 & 30156 & $\{11,13\}$ & 3 & 2 & 0.00006 & 116253 \\
\hline
\end{tabular}

Since $\log \left(x_{0}\right)<7 \times 10^{5}$ in each case, Theorem 2.1 is thus a consequence of Proposition 6.1 and direct computations for small values of $p^{k} x_{1}$.

\section{$9 \quad$ The proof of Theorem 1.1}

The argument of Ecklund, Eggleton, Erdős and Selfridge [5] to deduce an analogue of Theorem 1.1 for $k \notin\{3,5,7\}$ depends essentially on the fact that, for values of $k>2$ outside this exceptional set, we always have $k \geq 2 \pi(k)$. Ignoring the contribution to $V$ of terms of the shape $n-i_{p}$, where $i_{p}$ is chosen (not necessarily uniquely) so that $p$ divides $n-i_{p}$ maximally, we are led to the conclusion that

$$
V>\frac{(n-k+1)^{k-\pi(k)}}{k !},
$$

which, for fixed $k \notin\{3,5,7\}$ and suitably large $n$ leads to the desired conclusion (for $k \in\{4,6,8\}$, one needs to argue somewhat more carefully). For $k \in\{3,5,7\}$, however, $k<2 \pi(k)$ and hence we cannot estimate trivially the contributions to $V$ coming from the terms $n-i_{p}$. Indeed, our proof of Theorem 1.1 requires an appeal to Theorem 2.1 with

$$
(p, q) \in\{(2,3),(2,5),(3,5),(5,7)\} .
$$


We begin by treating the case $k=5$. Let us suppose that there exists an integer $n \geq 10$ and nonnegative integers $\alpha, \beta, \delta$ and $V$ such that

$$
\left(\begin{array}{l}
n \\
5
\end{array}\right)=2^{\alpha} 3^{\beta} 5^{\delta} \cdot V \text { with } \operatorname{gcd}(V, 30)=1
$$

and

$$
V^{2}<\left(\begin{array}{l}
n \\
5
\end{array}\right)
$$

We will suppose further that $n>10004$; a direct computation shows that otherwise $n \in\{10,12,28\}$. We claim that there exist distinct integers $i, j, k \in$ $\{0,1,2,3,4\}$ such that

$$
n \equiv i(\bmod 8), n \equiv j(\bmod 9) \text { and } n \equiv k(\bmod 5) .
$$

If not, then one of the following holds :

$$
\begin{aligned}
& \text { (i) } \quad \nu_{2}(n(n-1)(n-2)(n-3)(n-4)) \leq 4, \\
& \text { (ii) } \nu_{3}(n(n-1)(n-2)(n-3)(n-4)) \leq 2,
\end{aligned}
$$

or there exists an $i \in\{0,1,2,3,4\}$ such that one of

$$
\text { (iii) } n \equiv i(\bmod 72), n \equiv i(\bmod 40) \text { or } n \equiv i(\bmod 45) \text {. }
$$

Here, by $\nu_{p}(m)$ we mean, for a positive integer $m$, the largest integer $t$ such that $p^{t}$ divides $m$. In cases (i) and (ii), arguing crudely, we have that

$$
V \geq \frac{(n-2)(n-3)(n-4)}{36}
$$

which, with (9.1) contradicts $n>10004$. In case (iii), we similarly have

$$
V \geq \frac{(n-2)(n-3)(n-4)}{24}
$$

again contradicting our lower bound upon $n$.

We may therefore assume the existence of distinct $i, j, k$ satisfying (9.2) and hence from

$$
n(n-1)(n-2)(n-3)(n-4)=2^{\alpha+3} 3^{\beta+1} 5^{\delta+1} V,
$$


write $n-i=2^{\alpha} n_{i}, n-j=3^{\beta} n_{j}$ and $n-k=5^{\delta+1} n_{k}$, for integers $n_{i}, n_{j}$ and $n_{k}$. It follows that

$$
V \geq n_{i} n_{j} n_{k}(n-3)(n-4) / 24
$$

Since

$$
\max \left\{\left|2^{\alpha} n_{i}-3^{\beta} n_{j}\right|,\left|2^{\alpha} n_{i}-5^{\delta+1} n_{k}\right|,\left|3^{\beta} n_{j}-5^{\delta+1} n_{k}\right|\right\} \leq 4,
$$

applying Theorem 2.1, we have that

$$
\max \left\{n_{i}, n_{j}\right\}>(n-4)^{0.285}, \max \left\{n_{i}, n_{k}\right\}>(n-4)^{0.25}
$$

and

$$
\max \left\{n_{j}, n_{k}\right\}>(n-4)^{0.216}, \quad \text { whence } n_{i} n_{j} n_{k}>(n-4)^{0.501} .
$$

Combining this with (9.3),

$$
V>\frac{(n-3)(n-4)^{1.501}}{24}
$$

which, with (9.1), implies the inequality $n<10^{341}$. Proposition 6.1 together with a simple computation based on the final sentence of this result implies a contradiction.

The case $k=7$ is similar. We consider the equation

$$
\left(\begin{array}{l}
n \\
7
\end{array}\right)=2^{\alpha} 3^{\beta} 5^{\delta} 7^{\gamma} \cdot V \text { with } \operatorname{gcd}(V, 210)=1
$$

where now we suppose that

$$
V^{2}<\left(\begin{array}{l}
n \\
7
\end{array}\right)
$$

As before, a routine computation ensures that $n \leq 10006$ implies that $n \in$ $\{21,30,54\}$. Assuming, then, that $n>10006$, it is easy to show, analogous to the case $k=5$, that necessarily there are distinct integers $0 \leq i, j, k, l \leq 6$ such that

$$
n \equiv i(\bmod 8), n \equiv j(\bmod 9), n \equiv k(\bmod 25) \text { and } n \equiv l(\bmod 7) \text {. }
$$

Thus, writing $n-i=2^{\alpha} n_{i}, n-j=3^{\beta} n_{j}, n-k=5^{\delta} n_{k}$ and $n-l=7^{\gamma+1} n_{l}$, we have

$$
\max \left\{\left|2^{\alpha} n_{i}-3^{\beta} n_{j}\right|,\left|5^{\beta} n_{k}-7^{\gamma+1} n_{l}\right|\right\} \leq 6
$$


and

$$
V \geq n_{i} n_{j} n_{k} n_{l}(n-4)(n-5)(n-6) / 720 .
$$

Applying Theorem 2.1, we obtain

$$
n_{i} n_{j} n_{k} n_{l}>(n-6)^{0.512}
$$

In fact, the only pair listed in that theorem with components greater than 10000 and difference $\leq 6$ is $(30618,30613)$, and 30613 has no prime divisor $\leq 7$ so this pair needn't be considered. Combining (9.4), (9.6) and (9.7) with $n<10^{168}$ results in a contradiction to Proposition 6.1 . This completes the proof of Theorem 1.1.

\section{The proof of Theorem 1.2}

Theorem 1.2 is an easy consequence of the following more general result.

Theorem 10.1. Let $x$ and $D$ be positive integers with $D \leq 100$ and $D$ coprime to 6 , and suppose that $k, l$, and $y$ are nonnegative integers, with $y$ coprime to 6 , satisfying

$$
x^{2}+D x=2^{k} 3^{l} y
$$

Then one of the following holds:

(i) $y \geq x^{0.285}$

(ii) $y=1$ and one of $x$ and $x+D$ is of the form $2^{k}$ with $k \leq 8$ and the other is of the form $3^{l}$ with $l \leq 5$

(iii) $(x, D)$ is either $(640,89)$ or $(32768,37)$.

The proof is almost an immediate consequence of Theorem 2.1. Since $D$ is coprime to 6 , at most one of $x$ and $x+D$ is divisible by 2 and at most one is divisible by 3 . We deduce that $y \geq x$ unless one of $x$ and $x+D$ is of the form $2^{k} x_{1}$ and the other is of the form $3^{l} x_{2}$ where $x_{1}$ and $x_{2}$ are positive integers coprime to 6 . Thus,

$$
\left|2^{k} x_{1}-3^{l} x_{2}\right| \leq 100 \quad \text { and } \quad y=x_{1} x_{2} \geq \max \left\{x_{1}, x_{2}\right\}
$$

If $x>1000$, then Theorem 2.1 implies that either $\left(2^{k} x_{1}, 3^{l} x_{2}\right)$ or $\left(3^{l} x_{2}, 2^{k} x_{1}\right)$ belongs to a set of 40 elements listed explicitly in the theorem. A check 
through these 40 elements and a direct computation of the $x \leq 1000$ establishes the theorem.

As a final note, it is perhaps worth mentioning that the restriction here to values of $D$ coprime to 6 is essentially for simplicity and can be removed (with a slight reduction in the exponent 0.285) after a short computation, noting two more exceptional pairs with $x>1000$, namely $(x, D)=(1458,78)$ and $(65536,74)$.

\section{References}

[1] M. Bauer and M. A. Bennett, Applications of the hypergeometric method to the generalized Ramanujan-Nagell equation, Ramanujan J. 6 (2002), no. 2, 209-270.

[2] M. Bennett, Fractional parts of powers of rational numbers, Math. Proc. Cambridge Philos. Soc. 114 (1993), no. 2, 191-201.

[3] M. Bennett, Rational approximation to algebraic numbers of small height; the Diophantine equation $\left|a x^{n}-b y^{n}\right|=1$, J. Reine Angew. Math. 535 (2001), 1-49.

[4] F. Beukers, Fractional parts of powers of rationals, Math. Proc. Cambridge Philos. Soc. 90 (1981), no. 1, 13-20.

[5] E.F. Ecklund, jr., R.B. Eggleton, P. Erdös and J.L. Selfridge, On the prime factorization of binomial coefficients, J. Austral. Math. Soc. 26 (1978), 257-269.

[6] P. Erdős, Some unconventional problems in number theory, Acta Mathematica Academiae Scientarum Hungaricae, Tomus 33 (1979), 71-80.

[7] M. Filaseta and R. L. Williams, Jr., On the irreducibility of a certain class of Laguerre polynomials, J. Number Theory 100 (2003), 229-250.

[8] R. Gow, Some generalized Laguerre polynomials whose Galois groups are the alternating groups, J. Number Theory 31 (1989), 201-207.

[9] R. Guy, Unsolved Problems in Number Theory, 3rd ed., Springer Verlag 2004. 
[10] D. H. Lehmer, On a problem of Störmer, Illinois J. Math. 8 (1964), $57-79$.

[11] K. Mahler, Lectures on Diophantine Approximations I, University of Notre Dame, 1961.

[12] O. Ramaré and R. Rumely, Primes in arithmetic progressions, Math. Comp. 65 (1996), 397-425.

[13] D. Ridout, The p-adic generalization of the Thue-Siegel-Roth theorem, Mathematika 5 (1958), 40-48.

[14] J. B. Rosser and L. Schoenfeld, Sharper bounds for the Chebyshev functions $\theta(x)$ and $\psi(x)$, Math. Comp. 29 (1975), 243-269.

[15] L. Schoenfeld, Sharper bounds for the Chebyshev functions $\theta(x)$ and $\psi(x)$, II, Math. Comp. 30 (1976), no. 134, 337-360.

[16] I. Schur, Gleichungen ohne Affekt, Sitzungsberichte der Preussischen Akademie der Wissenschaften, Physikalisch-Mathematische Klasse (1930), 443-449.

[17] I. Schur, Affektlose Gleichungen in der Theorie der Laguerreschen und Hermiteschen Polynome, Journal für die reine und angewandte Mathematik 165 (1931), 52-58.

[18] T.N. Shorey and R. Tijdeman, Prime factors of arithmetical progressions and binomial progressions, to appear.

[19] C. Stewart, A note on the product of consecutive integers, Topics in classical number theory, Vol. II (Budapest, 1981), Colloq. Math. Soc. János Bolyai 34, North-Holland, Amsterdam, 1984, 1523-1537.

[20] C. Störmer, Vid. Skr. I Math.-Natur. Kl. (Christiania) (1897).

[21] J.J. Sylvester, On arithmetical series, Messenger Math. 21 (1892), 1-19, $87-120$. 